\title{
A modulated closed form solution for quantitative susceptibility mapping - A thorough evaluation and comparison to iterative methods based on edge prior knowledge
}

\author{
Diana Khabipova ${ }^{\mathrm{a}}$, Yves Wiaux ${ }^{\mathrm{b}}$, Rolf Gruetter ${ }^{\mathrm{a}, \mathrm{c}, \mathrm{d}}$, José P. Marques ${ }^{\mathrm{d}, *}$ \\ a Laboratory for Functional and Metabolic Imaging, Ecole Polytechnique Fédérale de Lausanne, Lausanne, Switzerland \\ ${ }^{\mathrm{b}}$ Institute of Sensors, Signals \&' Systems, Heriot-Watt University, Edinburgh, UK \\ c Department of Radiology, University of Geneva, Geneva, Switzerland \\ d Department of Radiology, University of Lausanne, Lausanne, Switzerland
}

\section{A R T I C L E I N F O}

\section{Article history:}

Accepted 18 November 2014

Available online 22 November 2014

\section{Keywords:}

Quantitative susceptibility mapping

Effective transverse relaxation

Phase imaging Brain Tissue susceptibility

magnetic resonance imaging (MRI)

Modulated closed form solution (MCF)

\begin{abstract}
A B S T R A C T
The aim of this study is to perform a thorough comparison of quantitative susceptibility mapping (QSM) techniques and their dependence on the assumptions made. The compared methodologies were: two iterative single orientation methodologies minimizing the 12, 11TV norm of the prior knowledge of the edges of the object, one overdetermined multiple orientation method (COSMOS) and a newly proposed modulated closed-form solution (MCF). The performance of these methods was compared using a numerical phantom and in-vivo high resolution ( $0.65 \mathrm{~mm}$ isotropic) brain data acquired at $7 \mathrm{~T}$ using a new coil combination method. For all QSM methods, the relevant regularization and prior-knowledge parameters were systematically changed in order to evaluate the optimal reconstruction in the presence and absence of a ground truth. Additionally, the QSM contrast was compared to conventional gradient recalled echo (GRE) magnitude and R2* maps obtained from the same dataset. The QSM reconstruction results of the single orientation methods show comparable performance. The MCF method has the highest correlation ( $\operatorname{corr}_{\mathrm{MCF}}=0.95, \mathrm{r}^{2}{ }_{\mathrm{MCF}}=0.97$ ) with the state of the art method (COSMOS) with additional advantage of extreme fast computation time. The L-curve method gave the visually most satisfactory balance between reduction of streaking artifacts and over-regularization with the latter being overemphasized when the using the COSMOS susceptibility maps as ground-truth. R2* and susceptibility maps, when calculated from the same datasets, although based on distinct features of the data, have a comparable ability to distinguish deep gray matter structures.
\end{abstract}

(C) 2014 Elsevier Inc. All rights reserved.

\section{Introduction}

Phase imaging has shown over the last decade to offer a good contrast, both between and within brain tissues in respect to the conventional magnitude signal (Duyn et al., 2007; Rauscher et al., 2005) as well as veins and iron rich regions (Haacke et al., 2004). The effect observed in the phase is known to be non-local, it reflects the magnetic field induced by the tissues' magnetic susceptibility (Marques and Bowtell, 2005), which scales linearly with the increase of the fields strength (making it suitable at high field strengths).

Several studies have been performed on the origin of the susceptibility contrast with the main modulators being iron and myelin. Iron contributes to tissue contrast especially in the deep gray matter (globus pallidus, putamen and caudate) which has histologically derived high iron concentration showing good correlation with phase and susceptibility contrast

* Corresponding author at: Station 6, CH-1015 Lausanne, Switzerland. Fax: + 4121 6937960.

E-mail address: jose.marques@epfl.ch (J.P. Marques).
(Bilgic et al., 2012; Schweser et al., 2011; Wharton and Bowtell, 2010). The other proposed contributor to the phase contrast, particularly between white and gray matter, is myelin where pathological demyelination has shown a decreased phase contrast between gray and white matter (C. Liu et al., 2011; Lodygensky et al., 2012) and good correlation was found between myelination and phase contrast during development. (Lodygensky et al., 2012).

In addition to the non-local effects associated with magnetic susceptibility, the chemical shift of water affected by macromolecules has been proposed to influence the measured phase (Luo et al., 2010; Shmueli et al., 2011; Zhong et al., 2008). More recently it was proposed (He and Yablonskiy, 2009) and demonstrated (Luo et al., 2013; Wharton and Bowtell, 2013; Yablonskiy et al., 2012) that the microstructural compartmentalization in the organization of lipids on the cellular and subcellular level (e.g. lipids, proteins) has a dominant effect on the contrast observed between white and gray matter in phase imaging.

Nevertheless, despite the last two effects being ignored when doing quantitative susceptibility mapping (QSM), this technique has demonstrated remarkable robustness in the ability to map iron deposition in 
deep gray matter structures (Langkammer et al., 2012; Schweser et al., 2011; Wharton and Bowtell, 2013). However, this problem is known to be ill-posed, and many methodologies have been suggested in order to better condition this problem. To make the problem overdetermined, field maps of the object have to be measured with the object positioned in different orientations in respect to the magnetic field (Liu et al., 2009). This method is not practical for clinical studies, due to the increased measurement time and not applicable to subjects with reduced mobility. For these reasons many methods have been proposed using single orientation field maps together with additional regularization which can be broadly fitted in two classes: $(i)$ correction of the k-space regions responsible by the artifact; (ii) prior-knowledge based on assumptions of smoothness and boundaries of the resulting QSM in the real space.

In the first class can be found direct methods that modify the kernel in a certain region which are responsible for the ill-conditioned nature of QSM (Schäfer et al., 2009; Schweser et al., 2013; Shmueli et al., 2009; Wharton and Bowtell, 2010), and the iterative methods that only use prior knowledge or sparsity constraints (11 or TV norm) to reconstruct the ill-conditioned points while trusting the remaining k-space with (Schweser et al., 2012) or without different weighting in the transition regions (Wu et al., 2012). Alternatively, in the second approach (ii), the whole k-space is affected by the introduced prior knowledge. The susceptibility calculation can be done by minimizing the 12 norm in real space field generated by the susceptibility map and the measured field maps together with additional regularization based on prior knowledge with either the 12 norm (de Rochefort et al., 2010) (see 12 regularized single-orientation method) or the 11 norm (Kressler et al., 2010; T. Liu et al., 2011) (see 11 total variation denoising method). The prior information is extracted from the phase and magnitude maps assuming them to have similar edges of the underlying brain structure or simply assuming that natural images are sparse in some basis set. Recently, it was noted that this could be performed as a direct inversion when assuming smoothness of the susceptibility map (Bilgic et al., 2013) (see Modulated closed form solution).

The aim of the present study was to perform a thorough comparison of some of these methods (de Rochefort et al., 2010; Liu et al., 2009; T. Liu et al., 2011) and a newly proposed methodology dubbed modulated closed form (MCF) both in simulations and in in-vivo data. Particularly we accurately evaluate the impact of the prior information and of the regularization parameters and how their optimality can be evaluated in the absence of ground truth. Additionally, the susceptibility results were compared to $\mathrm{R}_{2}^{*}$ contrast in both the contrast between gray and white matter, deep gray matter and ability to detect multiple sclerosis lesions.

\section{Theory}

The magnetic susceptibility, $\chi$, describes the reaction of a material to the presence of an external magnetic field. The magnetic field perturbation $\delta \mathrm{B}$ generated by a distribution of small magnetic susceptibility under a constant external magnetic field aligned to the $\mathrm{z}$-direction, $\mathrm{B}_{0}$, is given by a convolution of $\chi$ with the projection of the dipole field along the z-direction, D (Marques and Bowtell, 2005; Salomir et al., 2003). In the Fourier domain this can be simplified into a simple local expression:

$\delta B(k)=D(k) \cdot \chi(k)$

Where $\mathrm{k}$ are the $\mathrm{k}$-space coordinates and the magnetic dipole kernel can be written in $\mathrm{k}$-space as

$D(k)=\frac{-k_{x} \sin \theta-k_{y} \cos \theta \sin \varphi-k_{z} \cos \theta \cos \varphi}{\|k\|}+1 / 3$

Where $\theta$ describes the angle of rotation around the $x$-axis and $\varphi$ the angle of rotation around the $y$-axis. These angles characterize the orientation of the externally applied magnetic field, $\mathrm{B}_{0}$, in respect to the $\mathrm{z}$-direction of the object.

The dipole kernel in k-space has zero elements located in two conical surfaces. These surfaces lie at the magic angle direction in respect to the main magnetic field orientation. This means that the same field perturbation can be generated by a large number of different susceptibility distributions. As a consequence the direct inversion of Eq. (1) is an ill posed problem and noise in the measured field, $\delta B(r)$, gets significantly amplified in $\mathrm{k}$-space regions close to the two surfaces, leading to streaking artifacts in the reconstructed susceptibility maps.

In the following subsections a detailed description of the methods evaluated to overcome the ill posed nature of QSM will be given.

\section{Multiple orientation method - COSMOS}

Calculation Of Susceptibility through Multiple Orientation Sampling (COSMOS) takes advantage from the observation that the zero surface of the dipole kernel rotates with the magnetic field orientation $\mathrm{B}_{0}$ (Liu et al., 2009; Marques and Bowtell, 2005). Hence the straightforward methodology to overcome the ill posed nature of QSM implies the measurement of the field perturbation with the object oriented in various directions in respect to $\mathrm{B}_{0}$ (Liu et al., 2009; Marques and Bowtell, 2005). The $\chi$ map can then be calculated iteratively using a least squares conjugate gradient algorithm that minimizes,

$\min _{\chi} \sum_{i=1}^{N}\left\|M\left(F^{H} D_{i}(k) F \chi(\mathrm{r})-\delta B_{i}(r)\right)\right\|_{2}^{2}$

where $\mathrm{D}_{\mathrm{i}}$ and $\delta B_{i}(r)$ denote the dipole kernel and field perturbation for a specific object position, $i$ indexes the multiple object orientations, $F$ represents the Fourier Transform. $\mathrm{M}$ is a spatial mask that represents the regions inside the brain and is further modulated by a weighting term that guarantees that the noise throughout the field is equalized.

\section{$l_{2}$ regularized single-orientation method}

In the case where it is only possible to measure the field perturbation with the object positioned along one single orientation, extra information has to be introduced in the process of calculating the $\chi$ map. It is fair to assume that (i) the $\chi$ maps vary smoothly within anatomical boundaries/different tissue regions and (ii) that the artifacts, which are caused by the missing information around the magic angles, have structured sharp edges which cannot be found in the corresponding magnitude image. Consequently, regularization based on the 12 norm of the gradient has been widely promoted to tackle this problem (de Rochefort et al., 2010). As both the magnitude and the phase image images (Schweser et al., 2012) are expected to have similar edges as the underlying susceptibility distribution, they can be used as additional information to avoid the smoothing of the $\chi$ distribution close to tissue boundaries.

The regularized single-orientation (RSO) method incorporates prior knowledge of the expected edges by solving the following minimization problem using a least-squares conjugate gradient algorithm

$\min _{\chi}\left\|M\left(F^{H} D(k) F \chi(\mathrm{r})-\delta B_{0}(r)\right)\right\|_{2}^{2}+\beta\left\|M M_{\nabla} \nabla \chi(\mathrm{r})\right\|_{2}^{2}$

where the first term minimizes the distance between the estimated and measured field and the second term is the regularization prior tuned by a parameter $\beta$. The regularization term is a pixel by pixel multiplication of gradient of the susceptibility by a mask, $M_{\nabla}$, containing prior information regarding the regions where the gradients along a Cartesian direction are expected $\left(M_{\nabla}=0\right)$ or not $\left(M_{\nabla}=1\right)$. Both the regularization parameter and the gradient mask definition have a strong impact on the calculated $\chi$ map, the calculation of the latter will be discussed in the methods sections. 


\section{$l_{1}$ total variation denoising method}

Alternatively, because the $\chi$ maps, as many other natural images, to have well defined sharp contours surrounding areas of constant signal, total variation priors $l_{1}$ methods have been proposed in literature (Bilgic et al., 2012; Kressler et al., 2010). $1_{1}$ norm minimization boosts sparse solutions with a small number of non-zero elements and represents a useful convex relaxation of the $l_{0}$ norm, which simply counts the number of signal coefficients (Lustig et al., 2007; Puy et al., 2012). To facilitate the convergence, similarly to what has been suggested in the previous section for the $\mathrm{l}_{2}$ method, a prior information mask including the edge information, can also be applied (Liu et al., 2012; T. Liu et al., 2011). The susceptibility map is reconstructed from the field map by solving a so-called total variation denoising, TVDN (Beck and Teboulle, 2009), problem consisting of minimizing the TV norm of $\chi$ (the 11 norm of the gradient) which is subject to the same data constraint as for the 12 regularization:

$$
\min _{\chi}\left\|M M_{\nabla} \nabla \chi(\mathrm{r})\right\|_{1} \quad \text { s.t. } \quad\left\|M\left(F^{H} D_{i}(k) F \chi(\mathrm{r})-\delta B_{i}(r)\right)\right\|_{2}^{2}<\varepsilon
$$

In this constrained minimization, it is assumed that the data consistency term follows a $\chi^{2}$ distribution. The value of the bound $\varepsilon$ is thus driven by the noise statistics and should be simply set to a high percentile, of about $99 \%$, of this distribution.

\section{Modulated closed form solution}

The closed-form (CF) solution described in (Bilgic et al., 2013) relies on the Tikhonov problem $\min _{\chi}\left\|\left(F^{H} D(k) F \chi(\mathrm{r})-\delta B_{0}(r)\right)\right\|_{2}^{2}+\beta\|\nabla \chi(\mathrm{r})\|_{2}^{2}$, which can be evaluated in closed form as $\chi(\mathrm{r})=\left(F^{H} D^{2}(k) F+\right.$ $\left.\beta \nabla^{H} \nabla\right)^{-1} F^{H} D(k) F \delta B_{0}(r)$. The gradient operator along a direction $i$ can be described as $\partial_{i}=F^{H} E_{i} F$, where $\mathrm{F}$ is Fourier Transform, $E_{i}$ is given by $E_{i}=1-e^{-2 \pi j k_{i} / N_{i}}$, and $\mathrm{k}_{\mathrm{i}}$ is the k-space coordinate along $i$ direction. Using the k-space representation of the gradient operator the closed form can be analytically formulated as $\chi(k)=\frac{D(k)}{D(k)^{2}+\lambda^{2} \sum_{i=1}^{n=3} E_{i}^{2}} \delta B(k)$. This method is extremely fast but, when compared to the previously described iterative methods, the application of the gradient regularization in the whole image (and k-space), gives rise to smoother $\chi$ maps. To overcome these limitations, a weighting in the k-space of the regularization term was introduced to ensure that the regularization is only applied on the ill-conditioned k-space points, where the dipole kernel is smaller than a given threshold, $\mathrm{n}_{t h}$. The final expression of the modulated closed-form (MCF) solution can be written as

$\chi(k)=\frac{D(k)}{D(k)^{2}+\lambda^{2} \Lambda(\mathrm{k})^{2} \sum_{i=1}^{n=3} E_{i}^{2}} \delta B(k)$,

where $D(k)$ is the k-space representation of the dipole kernel, $\lambda$ is a regularization parameter, and $\Lambda(\mathrm{k})$ is a weighting matrix defined as

$$
\left\{\begin{aligned}
\cos \left(\frac{\frac{\pi}{2} D(k)}{\mathrm{n}_{t h}}\right), & D(k)<\mathrm{n}_{t h} \\
0, & D(k)>\mathrm{n}_{t h}
\end{aligned}\right.
$$

\section{Methods}

\section{Numerical simulation phantom}

A 3-dimensional numerical simulation phantom consisting of $64 \times 16 \times 64$ pixels containing 7 cylinders with different magnetic susceptibilities (between 2 and 14 a.u.) was used to evaluate the reconstruction performance of the different quantitative susceptibility mapping methods. The field map was calculated by using Eq. (1) assuming a magnetic field aligned along the z-direction. Zero mean Gaussian noise was added to the numerical phantom (that was used as our magnitude image), and to the field map. The ratio between the power of the images and that of noise was set to 60 (high enough to consider that Gaussian noise could be added directly to the field map). For the numerical simulations a metric that was initially suggested in (de Rochefort et al., 2010) was used to create a continuous gradient mask (if abs $(\nabla$ Magn $)<n_{t h} \sigma_{\text {Magn }}, M_{\nabla}=1$, else $M_{\nabla}=n_{t h} \sigma_{\text {Magn }} / \nabla$ MagnM) and a binary mask (if $\left(\operatorname{abs}(\nabla\right.$ Magn $)<n_{\text {th }} \sigma_{\text {Magn }}, M_{\nabla}=1$, else $\left.M_{\nabla}=0\right)$.

\section{In vivo data}

\section{Data acquisition}

Three healthy volunteers ( 2 males and 1 female, mean age of $30 \pm 6$ years) and one multiple sclerosis patient were scanned according to a protocol approved by the local ethics committee.

Scans were performed on a 7 T MR scanner (Siemens, Erlangen, Germany) using a 32 channel receive coil (Nova Medical). The protocol consisted of a standard T1-w MP2RAGE contrast (Marques et al., 2010) and $\mathrm{T}_{2}{ }^{*}$-w imaging using $3 \mathrm{D}$ gradient echo multi echo sequence. The 5 acquired echoes were equally spaced and acquired with the same polarity gradients, the rewinding waveform was kept equal to the readout gradient wave form to ensure flow compensation between successive echoes. The following parameters: $\mathrm{TR} / \mathrm{TE}_{1} / \mathrm{TE}_{5}=42 / 4.97 / 37.77 \mathrm{~ms}$; bandwidth $(\mathrm{BW})=260 \mathrm{~Hz} / \mathrm{Px} ; \mathrm{FA}=10^{\circ}, \mathrm{FOV}=256 \times 192 \times 137 \mathrm{~mm}$, spatial image resolution $660 \mu \mathrm{m} \times 660 \mu \mathrm{m} \times 660 \mu \mathrm{m}$; iPAT $=2 \times 2$; $\mathrm{T}_{\mathrm{acq}}=11 \mathrm{~min}$. This protocol was performed only once for the MS patient while for the healthy volunteers the 3D-GRE sequence was repeated 4 times with different head positions: normal; head tilted around mediolateral axis(left-right axis, pitch) in head-to-neck direction (up to $14^{\circ}$ ) position; tilted around anterior-posterior (nose-neck axis, roll) in head-to-left-shoulder direction (up to $25^{\circ}$ ) and head-to-right-shoulder direction (up to $25^{\circ}$ ). The exact head rotations were determined by coregistering all volumes to the first head position FSL-FLIRT (www.fmrib. ox.ac.uk). For the co-registration protocol to cope with the large head rotations that resulted in large variations of the image intensity, a bias field correction was applied to all magnitude images using FSL-FAST. Subsequently, a first co-registration was conducted prior to brain extraction in order to achieve a rough alignment of the structural images. Brain extraction was then performed to the co-registered head positions. On the resulting brain extracted images an additional FSL-FLIRT co-registration was calculated to get a more accurate registration. The movement matrices of the first and second stage co-registration were combined and the resulting movement matrix was applied to the original head positions to minimize the effect of double smoothing from FSL-FLIRT.

\section{Data processing}

Coil combination. All data processing was performed in MATLAB (version 2010b, The MathWorks, Natick, MA, USA) on a workstation (2× Intel Xeon X5650) with 96 GB RAM. The multi-channel GRE data from the different coils was combined using a pixel by pixel SVD factorization of the channel vs. echo time matrix similarly to what has been proposed for spectroscopy (Bydder et al., 2008). The first singular value of the diagonal matrix is the maximum signal that can be coherently constructed from the 32 channels and the 5 echoes acquired, the corresponding eigenvectors are the 32 complex coil sensitivity estimations that produce the desired image and the complex signal evolution along the 5 echoes (see Appendix for more details). Because the analysis is done on a pixel by pixel basis, the phase is somewhat arbitrary but the phase differences between successive echoes are not. This methodology gives the optimum SNR both for the magnitude and phase evolution as the coil sensitivities are constructed using the whole dataset and not only the first echo. 
Field and $R_{2}^{*}$ map calculation. The GRE phase differences between successive echoes were then unwrapped with a 3D phase unwrapping algorithm (Abdul-Rahman et al., 2007) in order to put the least demand in terms of number of wraps the algorithm had to cope with (also, by using phase differences between successive echoes, the data is devoid of phase singularities). Four field maps were computed by integrating the phase differences from the first to echo $n(n=2: 5)$ and the final field map was calculated using the phase differences between each phase image and that of $\mathrm{TE}_{1}$, weighted as in (Gruetter, 1993) using a pixel by pixel $\mathrm{R}_{2}^{*}$ estimation. Because the magnitude images for each echo were computed by complex coil combination followed by taking the absolute part of the complex signal $\left(I m_{e c}\right)$, and not by simple sum of squares of the separate coils, the magnitude evolution is not biased by Rician noise to the same extent (see Appendix for a discussion). Hence, the $R_{2}^{*}$ maps can be robustly calculated by integration of the magnitude decay:

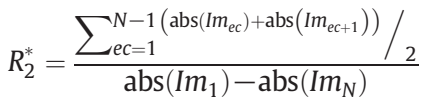

Background field removal. The measured field, $\delta \mathrm{B}$, inside the brain consists of the sum of internal variations, $\delta B_{i n}$, the mean brain susceptibility, $\mathrm{B}_{\text {mean }}$, and variations induced by external sources, $\delta \mathrm{B}_{\text {out }}$, such as air tissue interfaces and imperfect shimming. This background field $\left(\delta \mathrm{B}_{\text {out }}\right)$ was removed from the calculated field map using the recently proposed Efficient and Automated Harmonic Field Pre-Filtering (De Rochefort et al., 2013). As it is known that the Laplacians $(\Delta)$ of $\delta B_{\text {out }}$ and $B_{\text {mean }}$ are equal to $0, \delta B_{\text {in }}$ was calculated by solving the following minimization problem

$\min _{\delta \mathrm{Bin}}\left\|W_{\Delta}\left(\Delta \delta \mathrm{B}-\Delta \delta \mathrm{B}_{\text {in }}\right)\right\|$

with $\mathrm{W}_{\Delta}$ being a shrunk brain mask modulated by the SNR of the measured field map. This methodology has the advantage of reducing the erosion around the brain introduced by methods such as SHARP (Schweser et al., 2011) and is less prone to introducing artifacts due to phase errors in regions of low SNR.

Gradient mask calculation. The results obtained with the simulations suggested there was no added value from introducing the continuous gradient mask, therefore with the in vivo data only the binary mask was used. The in vivo gradient mask integrated information from the $\mathrm{R}_{2}^{*}$ and $\delta \mathrm{B}_{\text {in }}$ as suggested in recent studies (Schweser et al., 2012). Because the two data sets have different noise characteristics, the images were first wavelet denoised and the gradients of each image were calculated. The final mask was defined as (if $\left(a b s\left(\nabla R_{2}^{*}\right)<P_{\mathrm{abs}\left(\nabla R_{2}^{*}\right), n t h} A N D\right.$ $\left.a b s\left(\nabla \delta \mathrm{B}_{\text {in }}\right)<P_{a b s\left(\nabla \delta \mathrm{B}_{\text {in }}\right), n t h}\right), M_{\nabla}=1$, else $\left.M_{\nabla}=0\right)$, where $\mathrm{P}_{\text {nth }}$ represents the gradient corresponding to a percentile $\mathrm{n}_{\mathrm{th}}$. Although the two methodologies are not equivalent, they both reflect a variation of the number of points used to define the mask, so there should be a simple monotonic relationship between the two.

Susceptibility mapping and evaluation. Four different reconstruction algorithms were evaluated both for the numerical simulations and the in vivo data: $l_{2}$ minimization (Section 12 regularized single-orientation method), $l_{1}$ TV minimization (Section 11 total variation denoising method) and modulated closed-form solution (Section Modulated closed form solution). The COSMOS method (Section Multiple orientation method - COSMOS) was used to calculate the in vivo ground truth susceptibility map.

For all methods their the reconstruction parameters were varied systematically over a wide range ( $\beta$ for the $l_{2}, \varepsilon$ for $l_{1}$ TV minimization, and $\lambda$ for closed-form solution) as well as the masking defining parameter, $\mathrm{n}_{\mathrm{th}}$. The reconstruction performance of the methods in respect to the ground truth was computed as $\left\|\chi_{\text {recon }}-\chi_{\text {GroundTruth }}\right\|_{2} /\left\|\chi_{\text {GroundTruth }}\right\|_{2}$. The heuristic L-curve method (Hansen, 2000) was also evaluated as a possible mean to estimate the optimal reconstruction. This method consisted in the assessment of the data consistency term, $\left\|M\left(F^{H} D(k) F \chi(r)-\delta B_{0}(r)\right)\right\|_{2}^{2}$, as a function of the regularization term of each method while varying values of the respective regularization parameters. The optimal reconstruction was considered as the parameter set of largest curvature on the L-curve (maximum of second derivative) as done by (Bilgic et al., 2012).

To evaluate the impact of the regularization used on the various single orientation methods on the measured susceptibility, regions of interest were defined using fslview (www.fmrib.ox.ac.uk) on the following brain regions: GP - globus pallidus; $\mathrm{SN}$ - susbtantia nigra; $\mathrm{C}$ - caudate; RN - red nucleus; P - putamen; FM - forceps major; IFOF - inferior fronto-occipital fasciculus. Various regions of interest with changing susceptibility values were defined and masking of these regions was manually performed. The mean value is derived from first averaging voxels for each ROI within individual subjects and then averaging over three subjects. The error was calculated as the mean value over the different subjects of the standard deviation in each ROI.

\section{Results}

\section{Numerical simulation phantom}

To determine the influence of different parameters on the reconstruction quality of the susceptibility maps we compared the performance of the different algorithms on the simulated data set. When using a continuous prior for the $\mathrm{l}_{2}$ algorithm, the range of the regularization parameter $\beta$ was restricted to values in the range of one order of magnitude (Fig. 1a). When using a binary mask prior for the $\mathrm{l}_{2}$ algorithm, the range of acceptable regularization increased by a factor $\sim 10$, (Fig. 1b). Generally, the reconstruction quality of the susceptibility maps was higher using the binary mask as the prior information and the optimum $\beta$ value increases with the reduction of the threshold.

These observations were similar when using the $l_{1}$ denoising algorithm similar for the continuous and binary mask reconstructions (Figs. 1c and d), however, an increased independence on the parameter $\varepsilon$ was noted, provided the optimal threshold was achieved (marginally higher than for the $l_{2}$ method) and lower deviation from the ground truth was observed. This reconstruction quality was for both, $\mathrm{l}_{1}$ TV and $l_{2}$, algorithms less parameter dependent when using the binary mask, hence only this mask was used in the in vivo applications.

When setting the prior information to a high threshold (regularization was applied virtually on all pixels) lead only to a blurred reconstruction of the susceptibility in areas where the edges were not identified if the regularization parameter was not "correctly" defined (compare if and $\mathrm{g}$ for 12 method and $1 \mathrm{i}$ and $1 \mathrm{j}$ for 11 method). When using a low threshold lead to noise propagation in the reconstructed susceptibility maps (see Figs. 1h and k), implying that a priori information is required to ensure a good reconstruction quality as well as independence of the reconstruction from regularization parameter.

The modulated closed form method has reconstruction errors smaller than those obtained both with similar direct methods proposed by other groups (Bilgic et al., 2013; Schweser et al., 2013). Not surprisingly, the optimum results (and independence on the regularization parameters) are found when the regularization is limited to a region tightly positioned around the magic angle cones ( $\mathrm{nthr}=0.1-0.2$ ) as done in other k-space modulated iterative methods (Schweser et al., 2012; Wu et al., 2012).

\section{In vivo data}

Qualitative comparison of multiple orientation susceptibility and $R_{2}^{*}$ maps

Following the evaluation of the algorithms on simulated data, we evaluated their performance on 3D-GRE data obtained as described in 

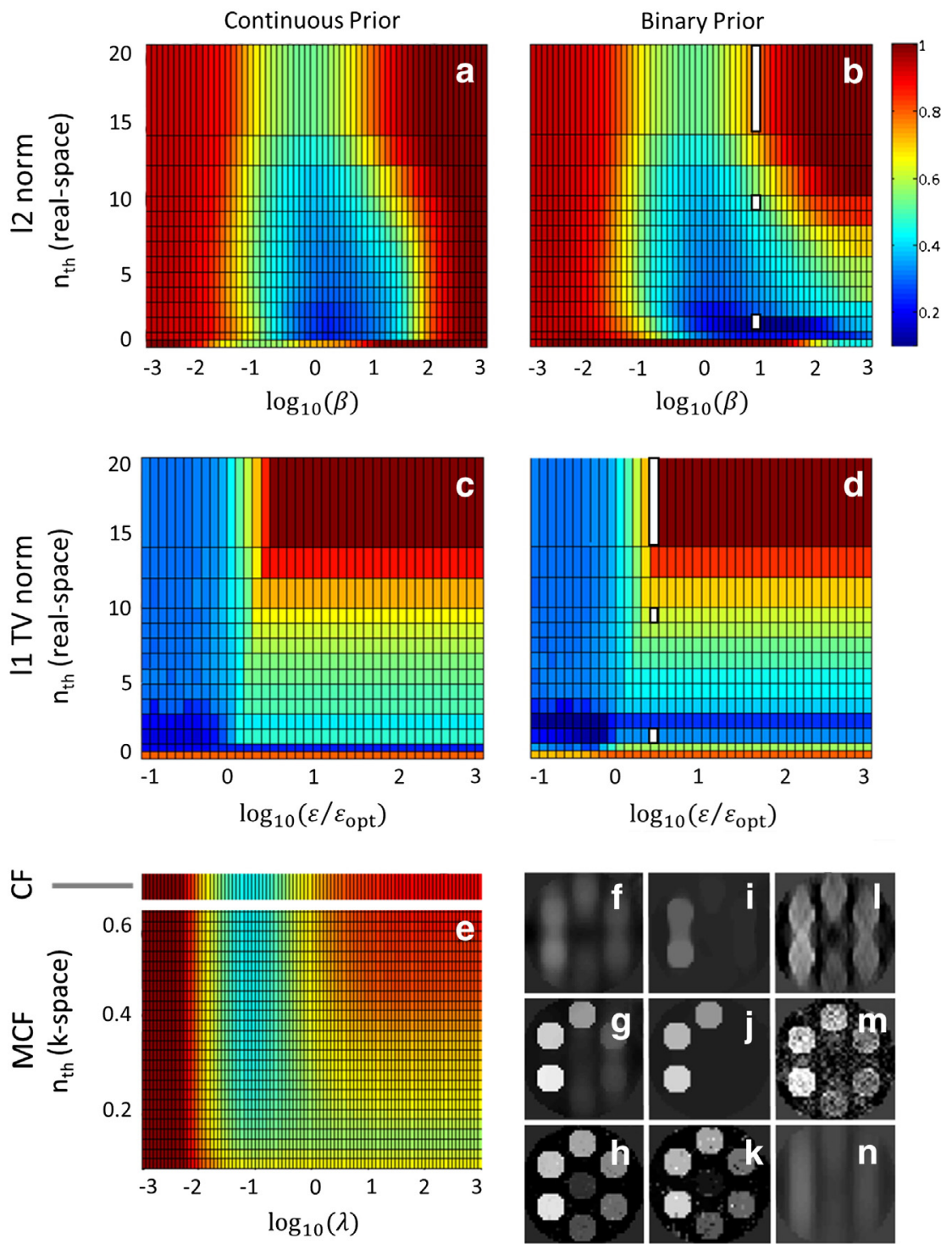

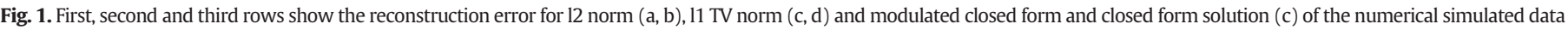

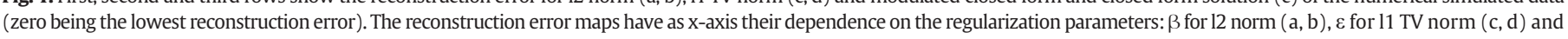

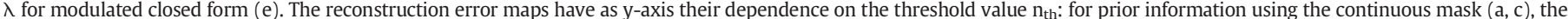

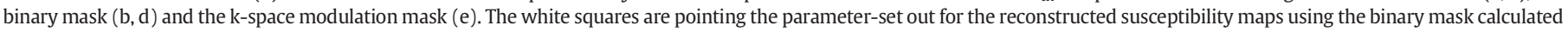

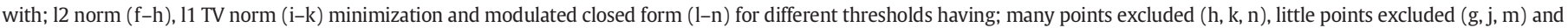
almost no points excluded (f, i, l) (low, high and very high threshold).

methods. The magnitude data obtained was computed after estimating the coil sensitivities that explain the maximum power of the signal over all echo times. As expected, the magnitude signal has high intensity variations associated with the use of the surface coil reception (the transmit coil effect is less clear). This methodology allows the calculation of very robust $\mathrm{R}_{2}^{*}$ maps over the whole brain which show both good vein delineation and deep gray matter contrast (thanks to the string magnetic susceptibility of de-oxygenated blood and iron) but also contrast between different white matter bundles (optic radian and the internal capsule among others are very clearly distinguishable) and gray white matter contrast (increase contrast is perceived on the frontal white matter in respect to the occipital and parietal). The background field removal quality from the complex data can be seen from the absence of large fields close to the boundaries together with the Laplacian of $\delta \mathrm{B}_{\text {out }}$ having no visible brain structure information. Note that this method allows most of the brain to be kept after background removal (including cortical regions parallel to the brain surface). The resulting high spatial resolution susceptibility maps obtained with COSMOS show the expected features described in other reports at $7 \mathrm{~T}$
(Deistung et al., 2013). In addition to deep gray matter regions, and the thalamic nuclei, white matter and significant variations of the contrast in different cortical regions were noted: e.g. the rim of increased para-magnetism of the frontal cortex noticeable at its white matter surface; increased para-magnetism of the occipital cortex in respect to the frontal cortex was evident from increased contrast. The latter is in agreement with previous reports increased transverse relaxation rates (decreased $\mathrm{T}_{2}$ ) in the occipital contrast in respect to both white matter and frontal cortex (Zhou et al., 2001).

\section{Single orientation susceptibility mapping methods}

To evaluate the reconstruction quality of the single orientation methods for a given regularization and threshold the power of the difference to the susceptibility maps was calculated with the COSMOS method, which was considered as the reference method, similarly to what was done for the numerical simulations. When using the 12 norm algorithm the optimum $\beta$ value increased with the reduction of the non-zero points (points where the regularization is effectively applied) present in the binary mask similar the numerical simulations. 
The reconstruction quality using the 11 algorithm shows good reconstruction using an optimal regularization parameter, although "better" results seem to be achieved using a binary mask with a high percentage of non-zero points unlike in numerical simulations. When using modulated closed form method optimal results were achieved when regularizing a relatively high region around the magic angle $\left(\mathrm{n}_{\mathrm{thr}}=0.3\right)$, as judged from the used weighting matrix in (Fig. 3 for e) broad and g) tight region around the magic angle). To verify the quality of the suggested optimal parameter set, susceptibility reconstructions for different parameter sets were compared (Fig. 4). After this initial evaluation all the remaining calculations of susceptibility maps shown throughout this manuscript were based on the following choice of parameters: the gradient weighting mask was defined with a $\mathrm{n}_{\mathrm{th}}=50 \%$; the modified closed form solution was obtained with $\mathrm{n}_{\text {th }}=0.2$. Therefore, the modified closed form solution was selected due to its ability to provide results that are largely independent from the regularization parameter.

Fig. 4 shows a small section of a coronal slice where the striking artifacts from the ill conditioned nature of susceptibility mapping originating from a large vein are clearly visible. The original phase measurement in those voxels is expected to have the largest errors, either due the low SNR of those voxels or due to incomplete flow compensation (only first order flow compensation was used). The impact of the regularization parameters for the different single orientation magnetic susceptibility methodologies can be observed both in the reduction of the striking artifacts and the intensity and separation between the different deep gray matter structures (and their intensities). When largely under-regularized solution was used, all methods exhibited large striking artifacts with the largest, unsurprisingly, being those associated with the CF and MCF methodology (left column of Fig. 4). Iterative methods are intrinsically regularized by the limited number of iterations. The QSM maps that suffers the most from over-regularization method are the 12 based methodology ( 12 norm and $\mathrm{CF}$ ) in which the values of all brain structures had reduced intensity due to the smoothness constraint as shown on the right column of Fig. 4. The 11 based method suffered less in the over-regularized regime in terms of intensity attenuation, with the over-regularization manifesting itself in the piece-wise smoothness of different white matter regions (right column of Fig. 4). The MCF method had the lowest impact of the over-regularization, although most striking artifacts have clearly disappeared. This was attributed to the use of a mask with a k-space smoothness term applied $\left(n_{\text {th }}=0.2\right)$ means that large sections of the $\mathrm{k}$-space suffer no regularization, in contrast to the $\mathrm{CF}$ method where the whole k-space is regularized. Surprisingly, many of these overregularized features can still be observed for regularization parameters that corresponded to a minimum deviation from the solution using the over-determined COSMOS method (solutions corresponding to colder colors in Figs. $2 \mathrm{a}-\mathrm{c}$ ). Using the heuristic L-curve point (represented in Figs. 2a-c by the black line) indicated solutions with reduced striking features where the intensity of the all deep gray matter structures was better preserved. For example, in the MCF image with the L-curve derived regularization (Fig. $4 \mathrm{j}$ ) it can be seen that the red nucleus has a sharper edge on its vertical orientation than on the oblique ones while in the CF solution (Fig. 4n) the all the boundaries appear smoother.

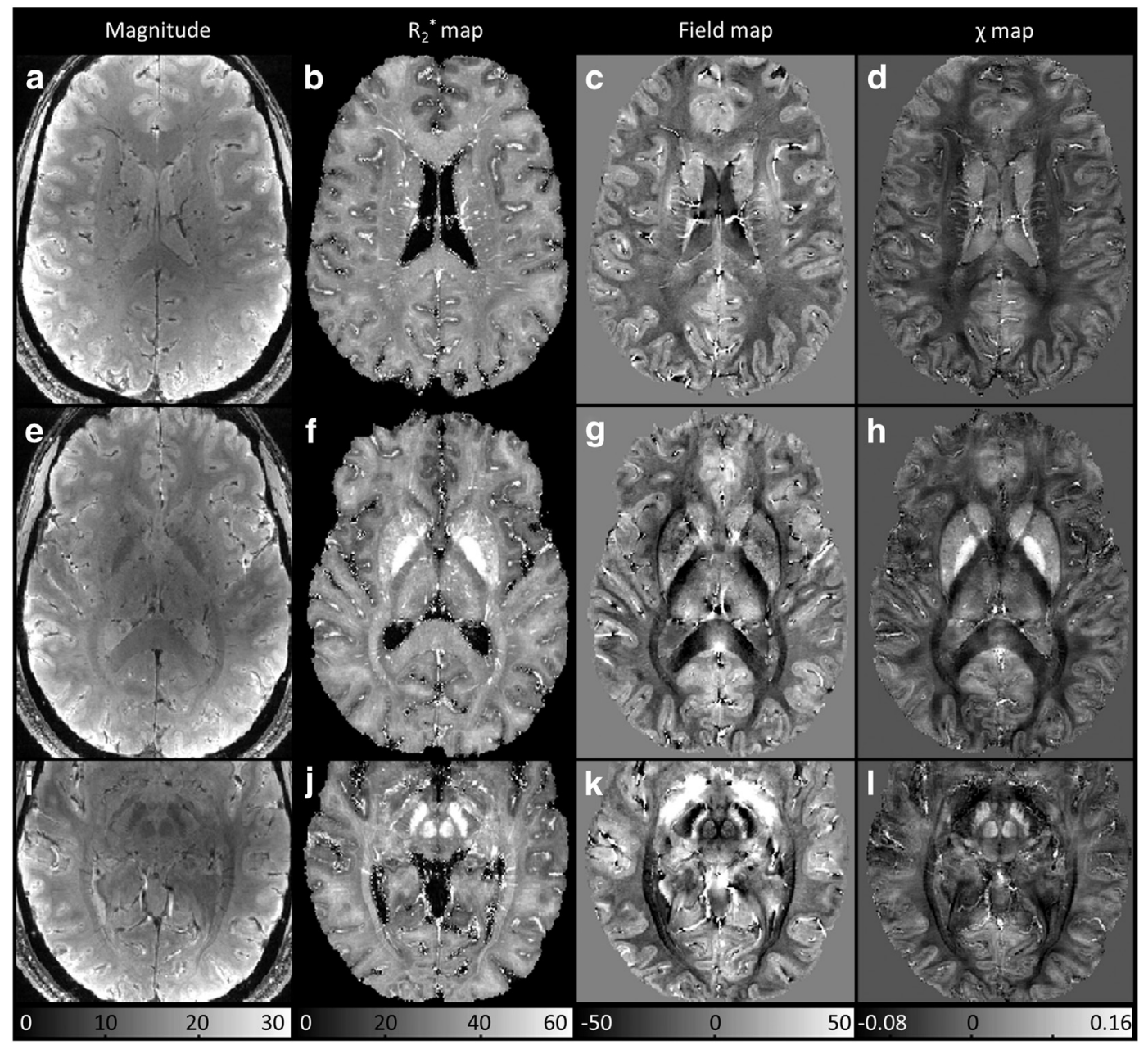

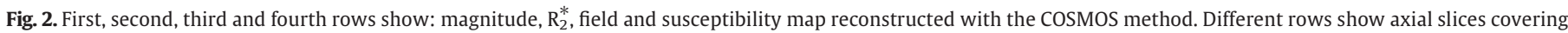
cortex (a-d); basal ganglia (e-h); substantia nigra (i-l). 

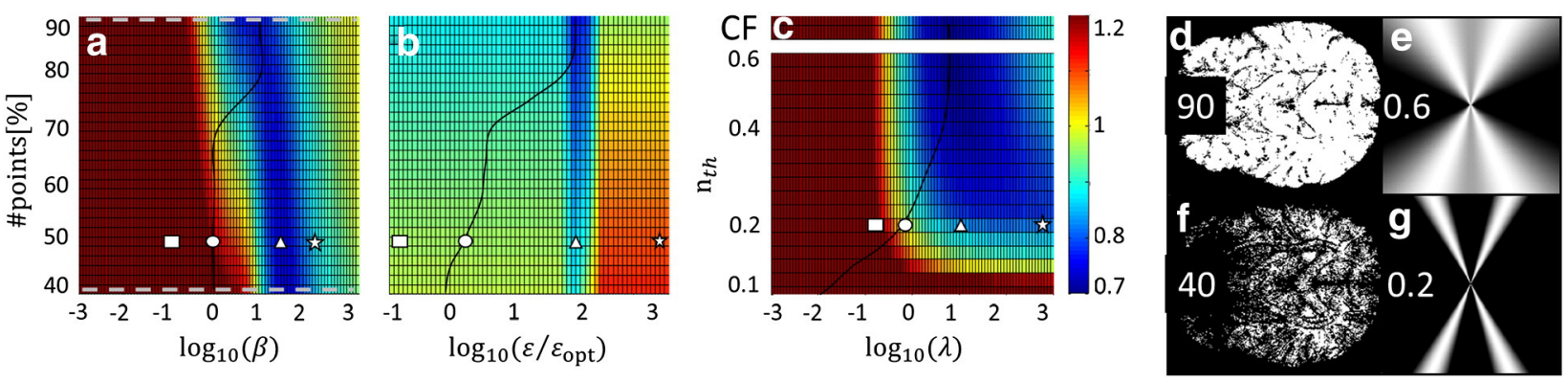

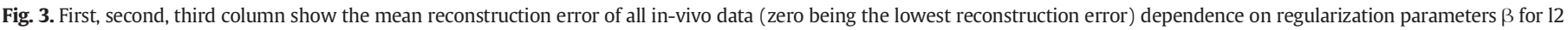

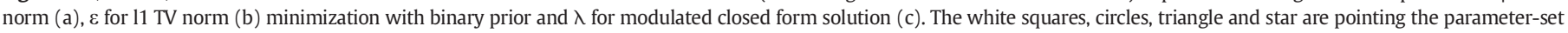

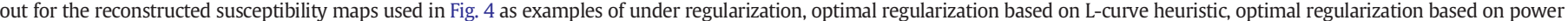

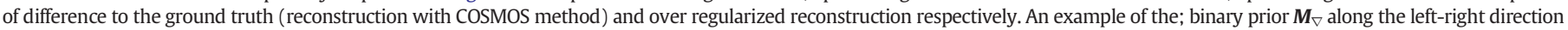

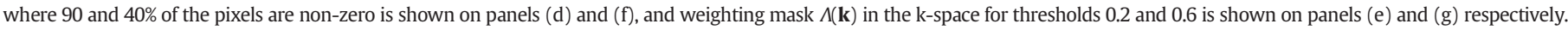

Fig. 5 shows the impact of different prior information mask, $M_{\nabla}$, for 12 norm and 11 TV norm minimization and weighting mask, $\Lambda(\mathbf{k})$, for modulated closed form solution. Applying $M_{\nabla}$, with a small number of non-zero points (40\%) on the single orientation methods (12 norm and 11 TV norm), leads to a regularization performed only on a limited number of pixels causing noise amplification (red arrows in Fig. 5). When the percentage of non-zero points is high (70\%), the regularization is performed everywhere, only excluding points with the strongest edge information, causing a blurred susceptibility map (Figs. 5c, f). In the case of the MCF method, a very tight weighting mask $\left(\left(\mathrm{n}_{t h}=0.1\right)\right)$, only a small number part of ill-conditioned points close to the magic angle cones were included in the regularization, causing noise related amplification artifacts (blue arrows in Fig. $5 \mathrm{~g}$ ). Green arrows point out the border between the substantia nigra and the red nucleus and the lamina pallidi, which can be easily distinguished using an optimal cone around the magic angle (nth $=0.2$ ), but not when applying the regularization on almost the whole k-space as done by the regular closed form solution (Figs. 5h, i).

High quality whole brain images could be obtained with the three single orientation methods using a regularization parameter obtained from the L curve analysis, although the best separation between the red nucleus and substantia nigra on the coronal slices was obtained with the COSMOS method (see Fig. 6). The COSMOS reconstruction also showed previously reported (Deistung et al., 2013) differentiable gray white matter contrast throughout the brain with a thin paramagnetic layer being observed in the frontal cortex in the GM-WM interface (highlighted with a yellow arrow). This layer is not so clearly observable on any of the single orientation methods $\chi$ maps. The red arrows show regions where the iterative single orientation methods ( 12 norm and 11 TV norm minimization) have noise amplification problems associated with regions where, due to the high gradients observed on the field map and $\mathrm{R}_{2}{ }^{*}$ maps, the gradient weighting terms, $M_{\nabla}$, had many contiguous points equal to zero, and hence no regularization was effectively applied. In this case the degree of prior knowledge of the susceptibility information effectively deteriorates the images reconstruction of these methods. The blue arrows highlight the white matter (optic radiation bundle) contrast observed throughout all the $\chi$ mapping methodologies. The green arrow shows the strong contrast and geometrical delineation of the cerebellum dentate nucleus that appears with lower intensity on the 11 TV norm minimization $\chi$ map but is otherwise successfully reconstructed.

\section{Quantitative comparison of susceptibility and $R_{2}^{*}$ mapping}

To compare the performance of the different $\chi$ reconstruction methods and $\mathrm{R}_{2}^{*}$ maps the correlation was evaluated in ROI in deep GM and WM. All single orientation methods (12 norm (red circle), 11 TV norm (green triangle) and MCF method (black square)) showed a linear correlation of the ROI with respect to the multiple-angle COSMOS method (see Fig. 7a) $\left(\operatorname{corr}_{11}=0.94, \mathrm{r}^{2}{ }_{11}=0.94, \operatorname{corr}_{12}=0.93, \mathrm{r}^{2}{ }_{12}=0.96\right.$, $\left.\operatorname{corr}_{\mathrm{MCF}}=0.95, \mathrm{r}^{2} \mathrm{MCF}=0.97\right)$. The regions with the highest standard deviation (substantia nigra and globus pallidus) show also the highest difference within the single orientation methods. When performing the

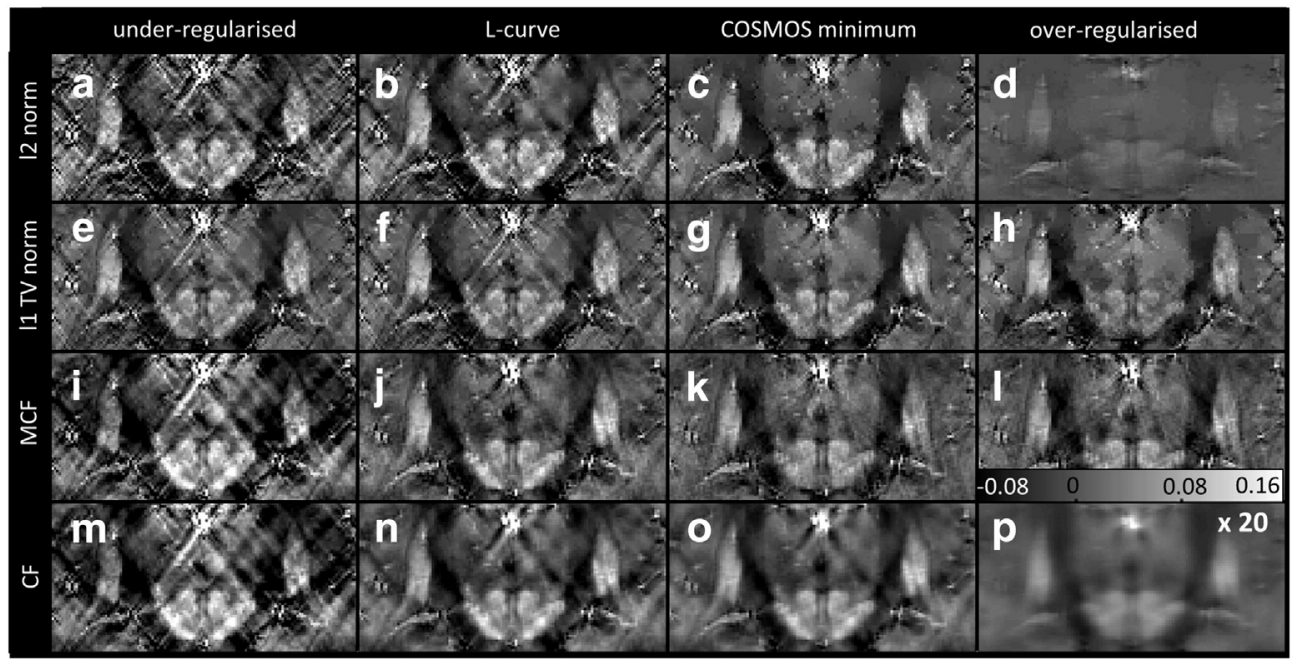

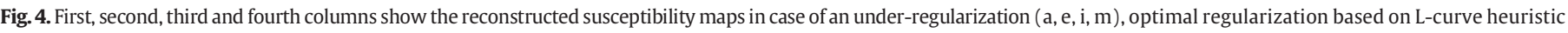

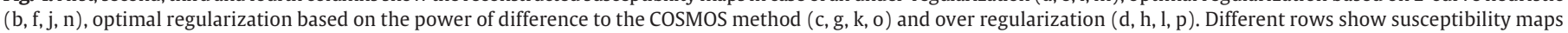
reconstructed with: 12 norm (a-d); 11 TV norm minimization (e-h), modulated closed-form solution (i-l) and closed-form solution (m-p). 


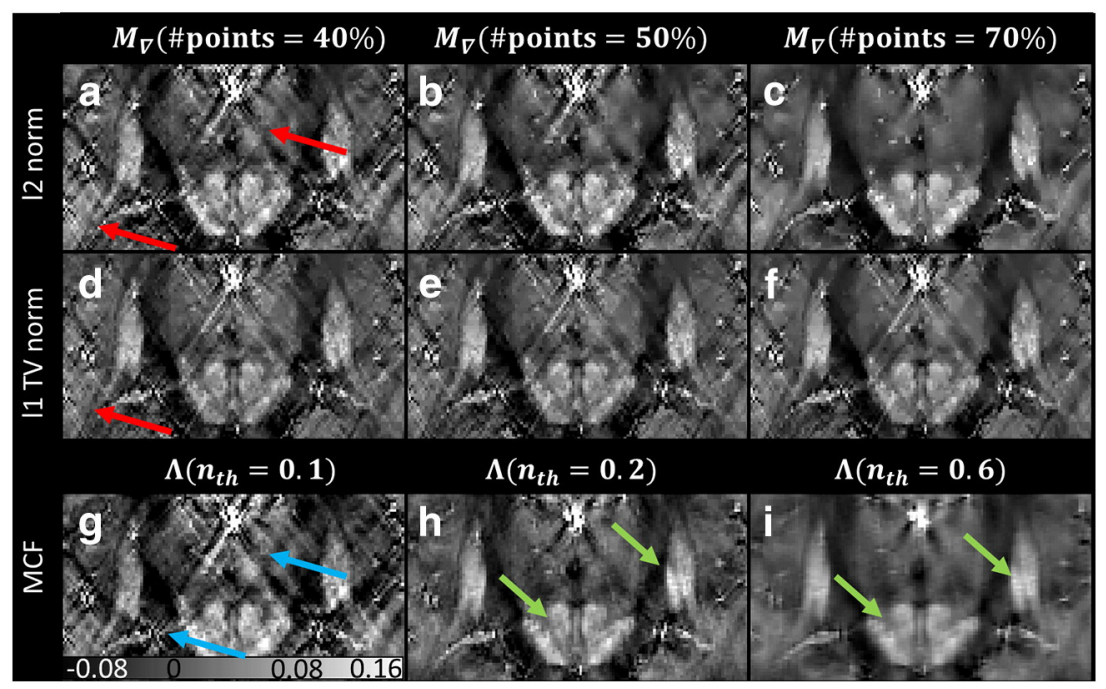

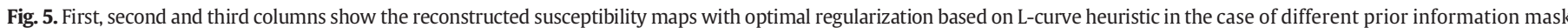

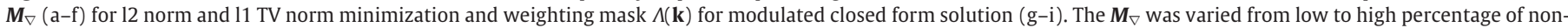

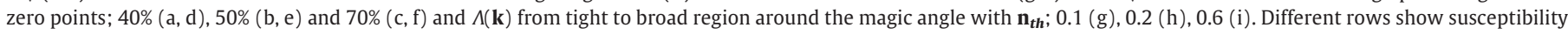
maps reconstructed with: 12 norm $(\mathrm{a}-\mathrm{c}) ; 11$ TV norm minimization $(\mathrm{d}-\mathrm{f})$ and modulated closed-form solution ( $\mathrm{g}-\mathrm{i})$.

same analysis as done in Fig. 7a using the single orientation methods of the remaining head positions, the substantia nigra had a tendency to be underestimated, yet the underestimation was always smaller than the variability observed in the ROI of the different subjects. Although a correlation could be found between $\mathrm{R}_{2}^{*}$ and $\chi$ values for the deep gray matter structures, it should be noted that for some of the deep gray matter structure significant $R_{2}^{*}$ variations were observed without a corresponding variations on susceptibility (Fig. 7b). The standard deviation found within each deep gray matter ROI in the $\mathrm{R}_{2}^{*}$ maps was of the order of magnitude of the difference between their mean values while for the susceptibility this ratio was increased. As reported in order studies, the difference between white matter and deep gray matter was significantly

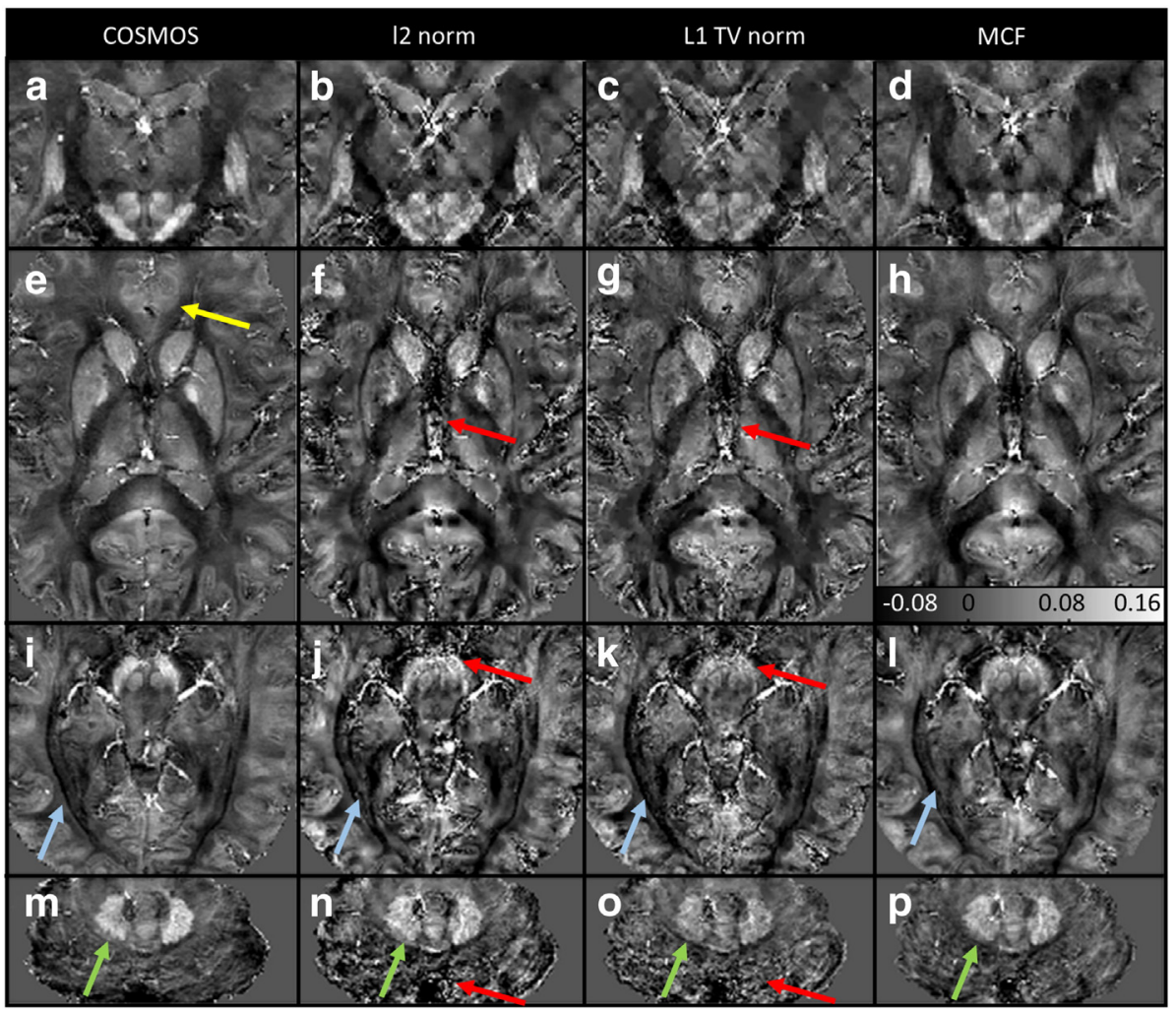

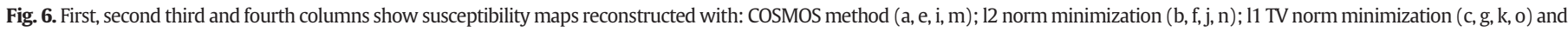

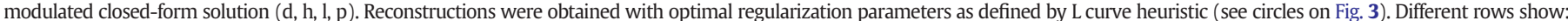

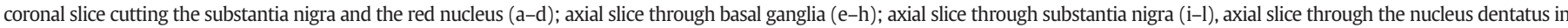

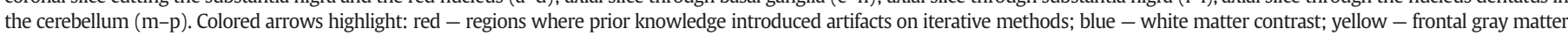
structures with enhanced subcortical contrast; green - structure of the nucleus dentatus. 

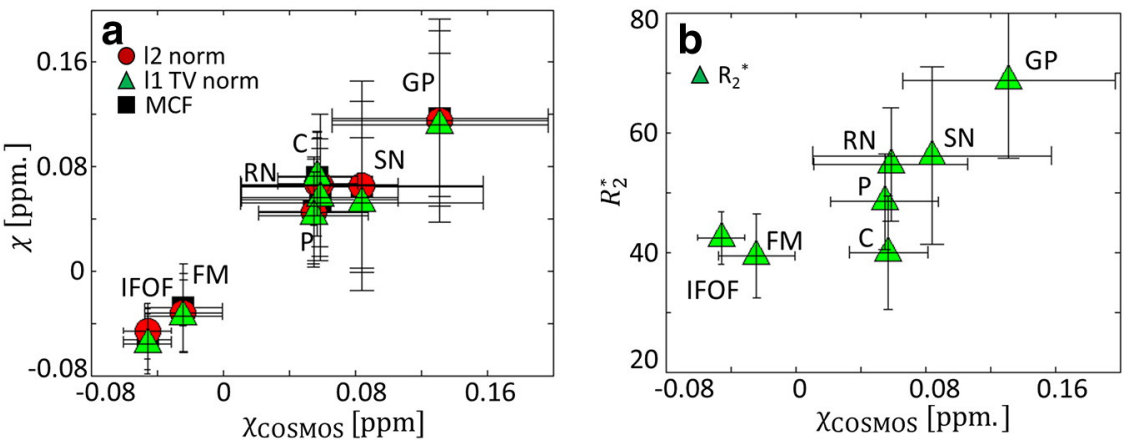

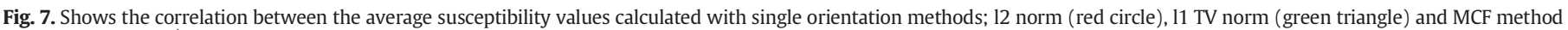

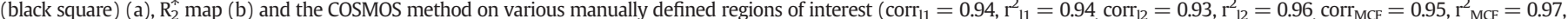

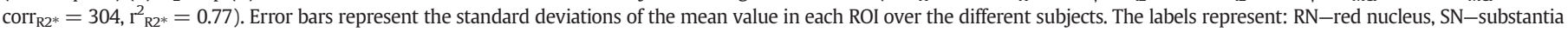
nigra, GP-globus pallidus, P-putamen, C-caudate, FM-forceps major, IFOF-inferior fronto-occipital fasciculus.

increased for the susceptibility (Deistung et al., 2013) which is to be expected given that the individual contributions of iron and myelin both tend to increase relaxation, whereas for susceptibility they have opposite behavior.

\section{Multiple sclerosis lesion detection}

The ability of $\chi$ maps to detect multiple sclerosis lesions was compared to conventional GRE acquisition contrasts (magnitude, $\mathrm{R}_{2}^{*}$, field) and MP2RAGE T1-weighted images. Fig. 8 shows a dataset from a multiple sclerosis patient where only one head orientation was acquired, and hence the COSMOS susceptibility map could not be calculated.

The green arrow points out a well-defined lesion with a positive contrast (increased paramagnetism) on all $\chi$ maps but that on the $\mathrm{R}_{2}^{*}$ map it shows a decreased relaxation rate while on the T1-w MP2RAGE image it appears dark, suggesting a very short longitudinal relaxation rate. The yellow arrow shows a lesion with a positive contrast on the magnitude images and susceptibility maps but on the $\mathrm{R}_{2}^{*}$ map it shows bright ring surrounding the lesion and the phase image shows a dark ring. The blue arrow highlights a lesion which appears diffuse both on the magnitude and the $\mathrm{R}_{2}^{*}$ maps (with lower relaxation rate), but appears better confined and with a positive contrast on the susceptibility maps and negative contrast on the T1-w images $\left(R_{1}\right.$ values similar to gray matter). The red arrow shows a lesion with well-defined positive contrast susceptibility and field maps but shows no contrast on the magnitude map.

\section{Discussion}

\section{Comparison of susceptibility and $R_{2}^{*}$ mapping}

For the first time, at $7 \mathrm{~T}$, a comparison of the sensitivity of $\mathrm{R}_{2}^{*}$ and $\chi$ maps calculated from exact the same data set was performed,

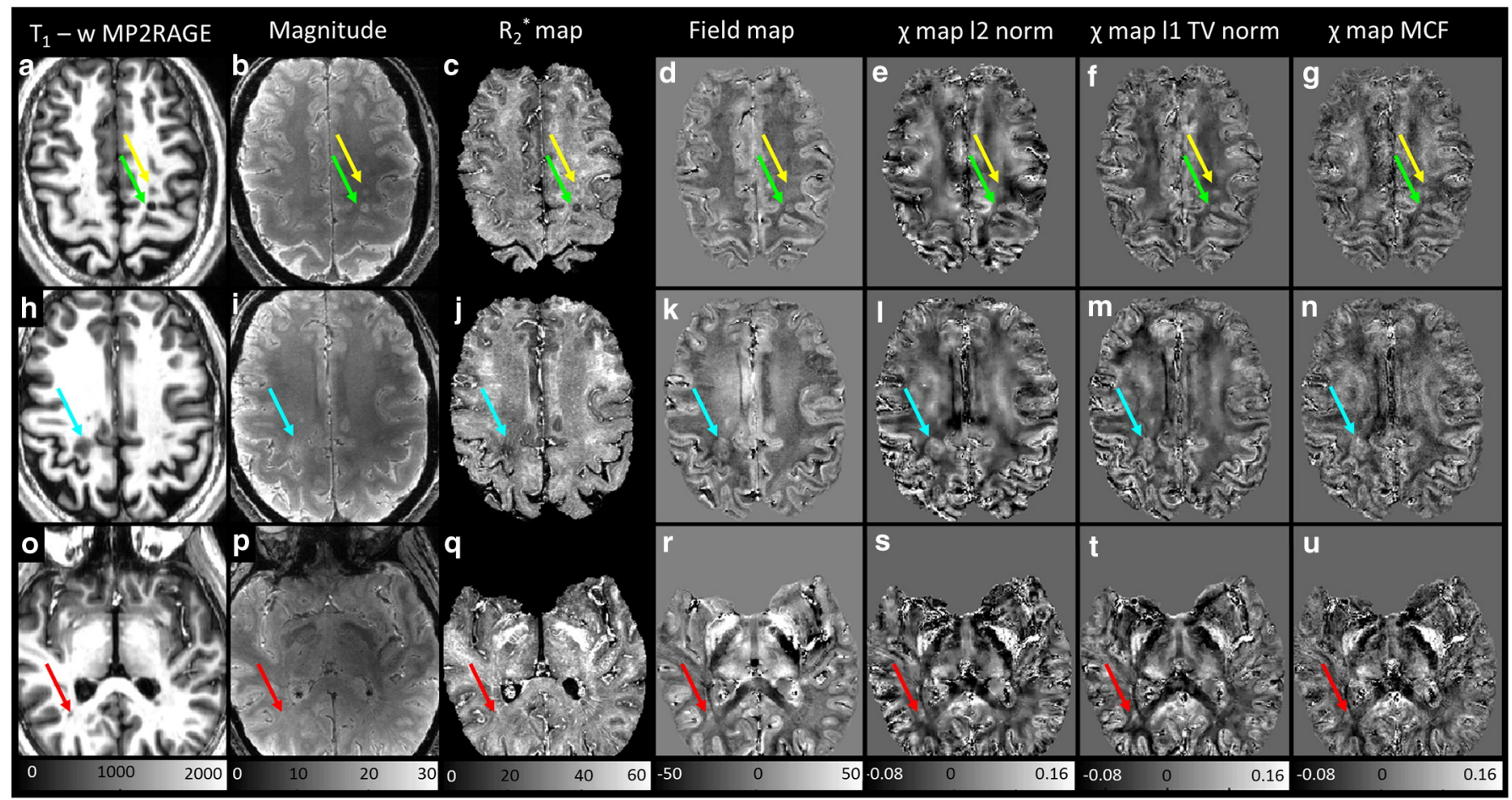

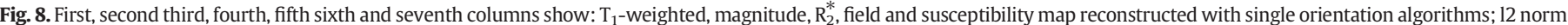

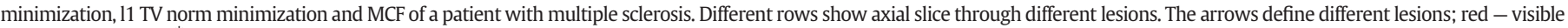

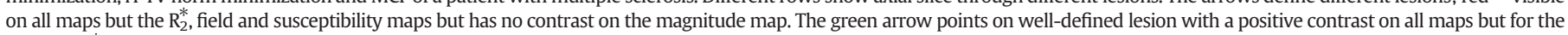
$\mathrm{T}_{1} \mathrm{~W}$ and $\mathrm{R}_{2}^{*}$ map. 
where all different orientations were used to create both the $\mathrm{R}_{2}^{*}$ and the susceptibility maps while in previous reports two different sequences and repetitions were used to retrieve $\mathrm{R}_{2}^{*}$ and susceptibility maps (Deistung et al., 2013). We conclude that using the proposed coil combination protocol, the $\mathrm{R}_{2}^{*}$ maps showed comparable or superior contrast to that shown by the susceptibility maps between the different deep gray matter regions. The biggest sensitivity of the susceptibility maps in respect to the $\mathrm{R}_{2}^{*}$ maps was found between white matter fiber bundles and between white and gray matter (even though recent findings suggest the observed contrast seen in white matter in phase images is wrongly attributed to the susceptibility (Luo et al., 2013)).

\section{Single orientation susceptibility mapping methods}

We performed first numerical simulations to evaluate the importance of the amount of prior knowledge. All methods benefited from having information from all possible boundaries in which case the result always showed the lowest distance to the ground truth and good results could be met for a large range of regularization parameters. It should be noted that the very piece wise constant nature of the phantom used in this numerical simulations benefited the performance of the iterative methods described that promote pice-wise constant or smooth solutions and are able to effectively denoise the reconstructed susceptibility map. This explains why the optimum modulated closed form solution had a significantly greater distance to the ground truth. The modified closed form solution was able to reduce the striking artifacts but the level of noise introduced in the field maps is maintained in the $\chi$ reconstructions (see Figs. 11-n).

When comparing the difference between multiple orientation and single orientation in vivo data, some small anatomical changes in the subcortical white matter in the frontal lobe (yellow arrow in Fig. 6) could be attributed both to: (a) the higher SNR associated with the implicit averaging performed by having 4 datasets contributing to the COSMOS reconstruction; (b) or increased artifacts of the single orientation methods; (c) or a forward model inconsistency associate with the microstructure; (d) inconsistent co-registration in the frontal lobe due to air tissue related artifacts. The last two are a plausible hypothesis because the subcortical layer is not clear on any of the individual field map images, and the fact that it gets reconstructed on the COSMOS, where the four datasets have to be physically consistent with one single susceptibility map ( $\left.\chi_{\text {cosmos }}\right)$ makes the methodology more sensitive both to the co-registration and the accuracy of the forward model. It was noticeable that the iterative methods exhibited increased noise in regions rich in both anatomical and phase contrast (see red arrow on Fig. 6). Because these regions were discarded from the mask, $M_{\nabla}$, the regularization did not affect them and therefore the ill-posed nature of the problem was emphasized. The discussed noise regions on the single orientation susceptibility maps corrupt the comparison to the COSMOS method. The effect of the noise regions with very high variability and standard deviation have a bigger effect than the over regularization artifacts when comparing single orientation methods to the COSMOS. Therefore the COSMOS method provided a less than optimal estimation and tended to favor over-regularized results. In the in vivo data, a good choice of the regularized information, either the prior information mask or ill-conditioned k-space region, rendered the susceptibility reconstruction methods highly independent of the regularization parameter. Under this scenario it was found that the L-curve heuristic method gave better results. As computing the l-curve is inherently computationally intensive having a fast method to perform the inversion can be an important asset. Throughout this paper the $11 \mathrm{TV}$ norm seemed to give the sharper anatomical results, yet this was at a cost of very large computation times ( $1 \mathrm{~h}$ per reconstruction) while the MCF solution could be calculated in a few seconds.

\section{Multiple sclerosis lesions}

It has been shown that tissue damage due to multiple sclerosis leads to the loss of macromolecules (myelin sheaths of the white matter axons) and therefore to a $\mathrm{R}_{2}^{*}$ hypo-intensities (negative contrast), reduction of the $\mathrm{R}_{2}^{*}$ relaxation rate (Yablonskiy et al., 2012), which were clearly visible (green and blue arrow in Fig. 8). The phase contrast changes are dependent from the underlying structural change; myelination damage could lead to hyper-intensities (positive contrast) while axonal damage could lead to hypo-intensities (negative contrast) (Yablonskiy et al., 2012), in our study all lesions showed a positive phase and susceptibility contrast. Moreover, in multiple sclerosis lesions where there are changes/ damage/injury of the myelin sheet, without loss of the tissue, could make them visible in the phase contrast map without being visible neither on the $R_{2}^{*}$ nor on the magnitude (Yablonskiy et al., 2012). This is the case of the lesion pointed with the red arrow which has been hypothesized to correspond mild or early multiple sclerosis lesions.

Various lesions could be easily detected in the $T_{1}-\mathrm{w}$ MP2RAGE data as reported by others (Kober et al., 2012). All detected lesion were visible in all the single orientation susceptibility reconstruction methods. However, some of the lesions are not as clearly defined on the 12 norm minimization as with the 11 TV norm minimization, which expects to have an underlying piece wise contrast. The susceptibility reconstructions with the modulated closed form solution have a lower SNR in respect to the iterative susceptibility reconstruction methods, but their SNR is comparable to that of the $\mathrm{R}_{2}^{*}$ map.

\section{Conclusion}

In this article we have implemented a fully flow compensated protocol for high spatial resolution GRE imaging at $7 \mathrm{~T}$ based on a multi-echo gradient echo sequence. The introduced combination of the multiple coil data both prevents phase singularities and maximizes the available SNR of the combination - this is done by implicitly using all the echo times to compute the receiving coil sensitivities. The proposed method to compute the field map is, from an error propagation perspective, the one that gives maximum SNR for the given echo times available (Gruetter, 1993). We have implemented different state-of-the-art methods to reconstruct susceptibility maps and performed a systematical analysis and comparison of the effects of the different regularization parameters and prior knowledge introduced. From the compared methodologies similar results were obtained with the highest correlation to the state of art methodology (COSMOS) being found for the MCF which has the additional advantage of being of extremely fast computationally.

We conclude that $\mathrm{R}_{2}^{*}$ and susceptibility have comparable quality to distinguish the deep gray matter structures while the susceptibility maps have a higher sensitivity to myelin related contrast, both in terms of white matter gray matter structures and early stage multiple sclerosis lesions.

\section{Acknowledgments}

This work was supported by Centre d'Imagerie BioMédicale (CIBM) of the UNIL, UNIGE, HUG, CHUV, EPFL and the Leenaards and Louis-Jeantet Foundations. The authors would also like to thank Cristina Cudalbu and Kieran O'Brien for providing the data set of a patient with multiple sclerosis lesions and Daniel Gallichan for support on the sequence development. D.K. and this project were funded by the Swiss National Science Foundation (SNF) grant no. 132821.

\section{Appendix A}

Numerical simulations were performed to evaluate the impact of the singular value decomposition (SVD) coil combination in the obtained quantitative maps. An exponential decay with a $\mathrm{T}^{*}=20 \mathrm{~ms}$ and 
frequency shift of $0.13 \mathrm{rad} / \mathrm{ms}$ was considered as our ground truth signal. The simulations assumed that $\mathrm{n}_{\text {echoes }}=5$ were acquired using $\mathrm{n}_{\text {coils }}=32$ (as in the experimental protocol).

The sensitivities of the 32 coils were created using a random complex number generator in order to take into account the different sensitivities of each coil, $C_{\text {coil }}$ (which in real data depends to a first order on the distance to the pixel of interest) and also to take into account the unknown receiving phase. Gaussian distributed complex noise was added to each channel and echo time. Resulting on

$S($ echo, coil $)=C_{\text {coil }} \times e^{\left(i \Delta \omega+1 / T_{2}^{*}\right) t_{\text {echo }}}+n$.

The noise, n,was introduced so that, in the case where the coil sensitivities were known, the mean SNR after combining the 32 channels over the 5 echoes was either 100 or 22.

The matrix $\mathrm{S}$ (with $\mathrm{n}_{\text {coils }}$ for column and $\mathrm{n}_{\text {echoes }}$ rows) was factorized using singular value decomposition and the vectors corresponding to the first singular value were kept. The left and right eigenvectors correspond to normalized relative coil sensitivities and the normalized time course. Alternatively, the magnitude time course was calculated by sum of squares combination (SOS) of the different coils. The field map calculation was done as described in the methods section (using the
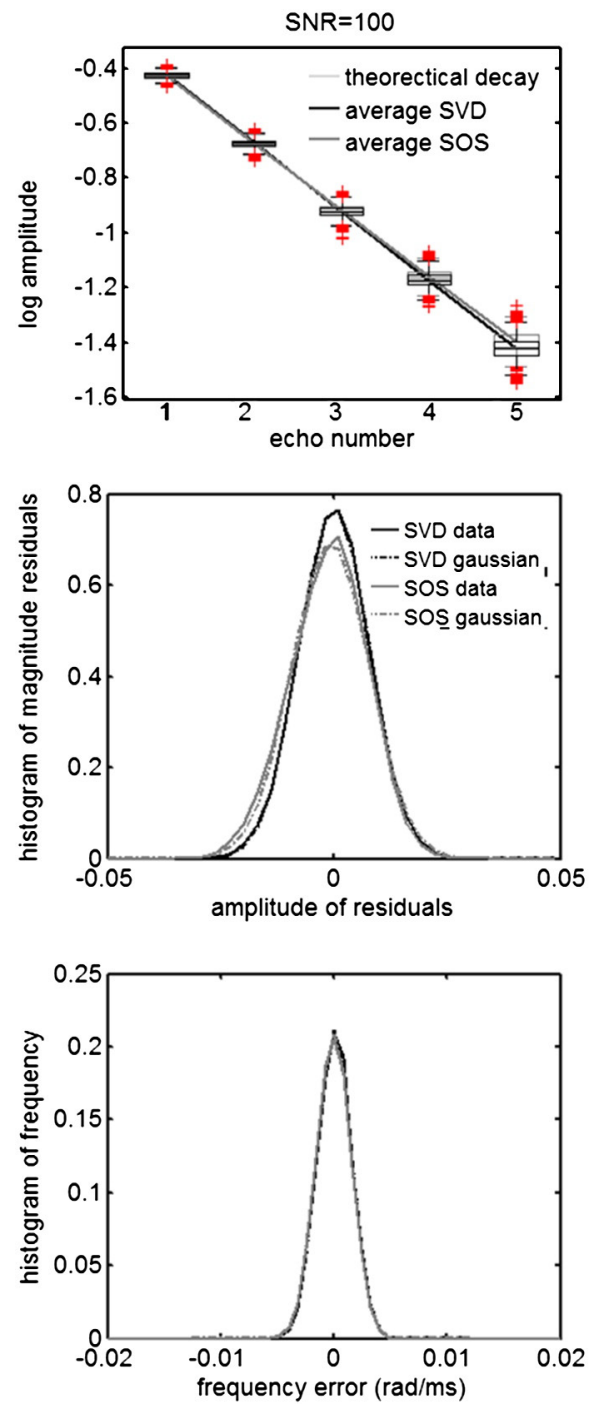

SNR weighting associated with a given echo time) but, while for the SVD time course it was computed only once, for the SOS methodology the fieldmaps were calculated independently for each channel and these were then combined using the power of each given channel as a weighting. This process was repeated 5000 times.

Fig. A1 shows on the first row that the SVD time course remains closer to the simulated signal. The reason for this discrepancy is likely due to the presence of channels that are noise dominated (which is also the case when using coil arrays with small loops). This deviation is larger for lower SNRs (see Fig. A1.b) which will contribute to an overestimation of the relaxation time (especially if doing the fit in the logarithmic scale). When fitting the simulated signal to the magnitude data as computed by SVD or by the SOS and plotting the residuals obtained over the 5000 iterations (see Fig. A1.d) it is possible to observe that: the SVD residuals have a smaller spread than the SOS (although for higher SNR values $>150$ they converged); the SVD residuals keep their Gaussian shape up to much lower SNRs (see Fig. A1.d). With the parameters used in the simulation the Gaussian shape of the SVD residuals was kept down to an SNR of 5 although from an SNR $<10$ deviations from the expected signal decay could be observed (looking at the magnitude images in Fig. 1, it can be seen estimated that such low SNR is only present in regions around veins but not in tissue). When looking at
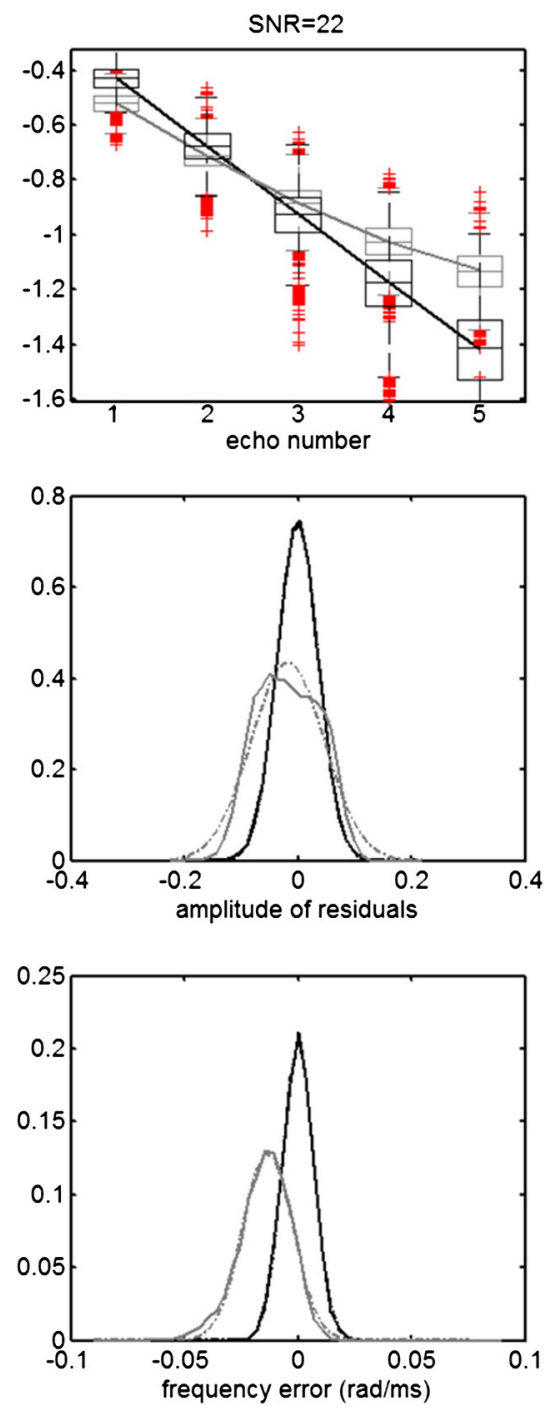

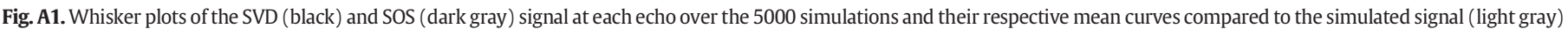

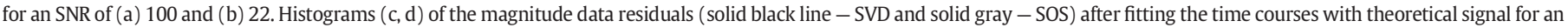

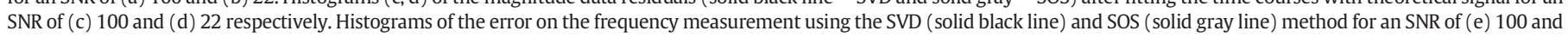
(f) 22 respectively. 
the impact of the SVD combination prior to the frequency calculation (see Figs. A1e and f), it can be seen that such a combination renders the calculation both more precise and accurate. The negative shift observed in the SOS frequency error at SNR $=22$ (see Fig. A1.f) results from the fact that in noise dominated channels the calculated frequency shift will be on average the zero. Despite making a weighted combination of the different channels, the underestimation propagates to the final SOS field maps.

It should be noted that some vendors already offer solutions with similar properties for the complex data by using a low resolution coil sensitivity prior scan. The SVD method has the particularity advantage of creating data driven normalized coil sensitivities that have the feature of generating the maximum signal as has been demonstrated by other groups for spectroscopy (Bydder et al., 2008). Such a method could also find applicability in, for example, DTI where, instead of various echoes, various contrasts exist with different degrees of SNR. Reducing the noise propagation in the low SNR measurements would allow a better accuracy of the measured diffusion parameters.

\section{References}

Abdul-Rahman, H.S., Gdeisat, M.A., Burton, D.R., Lalor, M.J., Lilley, F., Moore, C.J., 2007. Fast and robust three-dimensional best path phase unwrapping algorithm. Appl. Opt. 46, 6623-6635.

Beck, A., Teboulle, M., 2009. Fast gradient-based algorithms for constrained total variation image denoising and deblurring problems. IEEE Trans. Image Process. 18, 2419-2434. http://dx.doi.org/10.1109/TIP.2009.2028250.

Bilgic, B., Pfefferbaum, A., Rohlfing, T., Sullivan, E.V., Adalsteinsson, E., 2012. MRI estimates of brain iron concentration in normal aging using quantitative susceptibility mapping. Neurolmage 59, 2625-2635. http://dx.doi.org/10.1016/j.neuroimage.2011.08.077.

Bilgic, B., Chatnuntawech, I., Fan, A.P., Setsompop, K., Cauley, S.F., Wald, L.L., Adalsteinsson, E., 2013. Fast image reconstruction with L2-regularization. J. Magn. Reson. Imaging http:// dx.doi.org/10.1002/jmri.24365 (n/a-n/a).

Bydder, M., Hamilton, G., Yokoo, T., Sirlin, C.B., 2008. Optimal phased-array combination for spectroscopy. Magn. Reson. Imaging 26, 847-850. http://dx.doi.org/10.1016/j.mri.2008. 01.050 .

De Rochefort, L., Liu, T., Kressler, B., Liu, J., Spincemaille, P., Lebon, V., Wu, J., Wang, Y., 2010. Quantitative susceptibility map reconstruction from MR phase data using Bayesian regularization: validation and application to brain imaging. Magn. Reson. Med. Off. J. Soc. Magn. Reson. Med. Soc. Magn. Reson. Med. 63, 194-206. http://dx.doi.org/10.1002/ mrm.22187.

De Rochefort, Ludovico, Wand, Hongchen, Loureiro de Sousa, Paulo, Armspach, Jean-Paul, 2013. Efficient and automatic harmonic field pre-filtering for brain quantitative susceptibility mapping. ISMRM. Presented at the Annual Meeting of the International Society for Magnetitic Reasonance in Medicine, Salt Lake City, Utah, USA, p. 170.

Deistung, A., Schäfer, A., Schweser, F., Biedermann, U., Turner, R., Reichenbach, J.R., 2013. Toward in vivo histology: a comparison of quantitative susceptibility mapping (QSM) with magnitude-, phase-, and R2*-imaging at ultra-high magnetic field strength. Neurolmage 65, 299-314. http://dx.doi.org/10.1016/j.neuroimage.2012.09.055.

Duyn, J.H., van Gelderen, P., Li, T.-Q., de Zwart, J.A., Koretsky, A.P., Fukunaga, M., 2007. High-field MRI of brain cortical substructure based on signal phase. Proc. Natl. Acad. Sci. U. S. A. 104, 11796-11801. http://dx.doi.org/10.1073/pnas.0610821104.

Gruetter, R., 1993. Automatic, localized in vivo adjustment of all first- and second-order shim coils. Magn. Reson. Med. Off. J. Soc. Magn. Reson. Med. Soc. Magn. Reson. Med. 29, 804-811.

Haacke, E.M., Xu, Y., Cheng, Y.-C.N., Reichenbach, J.R., 2004. Susceptibility weighted imaging (SWI). Magn. Reson. Med. Off. J. Soc. Magn. Reson. Med. Soc. Magn. Reson. Med. 52, 612-618. http://dx.doi.org/10.1002/mrm.20198.

Hansen, P.C., 2000. The L-curve and its use in the numerical treatment of inverse problems. In: Johnston, P. (Ed.), In Computational Inverse Problems in ElectrocardiologyAdvances in Computational Bioengineering. . WIT Press, pp. 119-142.

He, X., Yablonskiy, D.A., 2009. Biophysical mechanisms of phase contrast in gradient echo MRI. Proc. Natl. Acad. Sci. U. S. A. 106, 13558-13563. http://dx.doi.org/10.1073/pnas. 0904899106.

Kober, T., Granziera, C., Ribes, D., Browaeys, P., Schluep, M., Meuli, R., Frackowiak, R., Gruetter, R., Krueger, G., 2012. MP2RAGE multiple sclerosis magnetic resonance imaging at $3 \mathrm{~T}$. Invest. Radiol. 47, 346-352. http://dx.doi.org/10.1097/RLI.0b013e31824600e9.

Kressler, B., de Rochefort, L., Liu, T., Spincemaille, P., Jiang, Q., Wang, Y., 2010. Nonlinear regularization for per voxel estimation of magnetic susceptibility distributions from MRI field maps. IEEE Trans. Med. Imaging 29, 273-281. http://dx.doi.org/10.1109/ TMI.2009.2023787.

Langkammer, C., Schweser, F., Krebs, N., Deistung, A., Goessler, W., Scheurer, E., Sommer, K., Reishofer, G., Yen, K., Fazekas, F., Ropele, S., Reichenbach, J.R., 2012. Quantitative susceptibility mapping (QSM) as a means to measure brain iron? A post mortem validation study. NeuroImage 62, 1593-1599. http://dx.doi.org/10.1016/j.neuroimage. 2012.05.049.

Liu, T., Spincemaille, P., de Rochefort, L., Kressler, B., Wang, Y., 2009. Calculation of susceptibility through multiple orientation sampling (COSMOS): a method for conditioning the inverse problem from measured magnetic field map to susceptibility source image in
MRI. Magn. Reson. Med. Off. J. Soc. Magn. Reson. Med. Soc. Magn. Reson. Med. 61, 196-204. http://dx.doi.org/10.1002/mrm.21828.

Liu, C., Li, W., Johnson, G.A., Wu, B., 2011a. High-field (9.4 T) MRI of brain dysmyelination by quantitative mapping of magnetic susceptibility. NeuroImage $56,930-938$. http:// dx.doi.org/10.1016/j.neuroimage.2011.02.024.

Liu, T., Liu, J., de Rochefort, L., Spincemaille, P., Khalidov, I., Ledoux, J.R., Wang, Y., 2011b. Morphology enabled dipole inversion (MEDI) from a single-angle acquisition: comparison with COSMOS in human brain imaging. Magn. Reson. Med. Off. J. Soc. Magn. Reson. Med. Soc. Magn. Reson. Med. 66, 777-783. http://dx.doi.org/10.1002/mrm.22816.

Liu, T., Xu, W., Spincemaille, P., Avestimehr, A.S., Wang, Y., 2012. Accuracy of the morphology enabled dipole inversion (MEDI) algorithm for quantitative susceptibility mapping in MRI. IEEE Trans. Med. Imaging 31, 816-824. http://dx.doi.org/10.1109/ TMI.2011.2182523.

Lodygensky, G.A., Marques, J.P., Maddage, R., Perroud, E., Sizonenko, S.V., Hüppi, P.S. Gruetter, R., 2012. In vivo assessment of myelination by phase imaging at high magnetic field. Neurolmage 59, 1979-1987. http://dx.doi.org/10.1016/j.neuroimage.2011.09.057.

Luo, J., He, X., d'Avignon, D.A., Ackerman, J.J.H., Yablonskiy, D.A., 2010. Protein-induced water $1 \mathrm{H}$ MR frequency shifts: contributions from magnetic susceptibility and exchange effects. J. Magn. Reson. San Diego Calif. 1997 202, 102-108. http://dx.doi.org/10.1016/j. jmr.2009.10.005.

Luo, J., He, X., Yablonskiy, D.A., 2013. Magnetic susceptibility induced white matter MR signal frequency shifts-experimental comparison between Lorentzian sphere and generalized Lorentzian approaches. Magn. Reson. Med. Off. J. Soc. Magn. Reson. Med. Soc. Magn. Reson. Med. http://dx.doi.org/10.1002/mrm.24762.

Lustig, M., Donoho, D., Pauly, J.M., 2007. Sparse MRI: the application of compressed sensing for rapid MR imaging. Magn. Reson. Med. 58, 1182-1195. http://dx.doi.org/10. 1002/mrm.21391.

Marques, J.P., Bowtell, R., 2005. Application of a Fourier-based method for rapid calculation of field inhomogeneity due to spatial variation of magnetic susceptibility. Concepts Magn. Reson. Part B: Magn. Reson. Eng. 25, 65-78. http://dx.doi.org/10.1002/cmr.b. 20034.

Marques, J.P., Kober, T., Krueger, G., van der Zwaag, W., Van de Moortele, P.-F., Gruetter, R. 2010. MP2RAGE, a self bias-field corrected sequence for improved segmentation and T1-mapping at high field. Neurolmage 49, 1271-1281. http://dx.doi.org/10.1016/j. neuroimage.2009.10.002.

Puy, G., Marques, J.P., Gruetter, R., Thiran, J.-P., Van De Ville, D., Vandergheynst, P., Wiaux, Y., 2012. Spread spectrum magnetic resonance imaging. IEEE Trans. Med. Imaging 31, 586-598. http://dx.doi.org/10.1109/TMI.2011.2173698.

Rauscher, A., Sedlacik, J., Barth, M., Mentzel, H.-J., Reichenbach, J.R., 2005. Magnetic susceptibility-weighted MR phase imaging of the human brain. AJNR Am. J. Neuroradiol. 26, 736-742.

Salomir, R., de Senneville, B.D., Moonen, C.T., 2003. A fast calculation method for magnetic field inhomogeneity due to an arbitrary distribution of bulk susceptibility. Concepts Magn. Reson. Part B: Magn. Reson. Eng. 19B, 26-34. http://dx.doi.org/10.1002/cmr.b. 10083.

Schäfer, A., Wharton, S., Gowland, P., Bowtell, R., 2009. Using magnetic field simulation to study susceptibility-related phase contrast in gradient echo MRI. NeuroImage 48 126-137. http://dx.doi.org/10.1016/j.neuroimage.2009.05.093.

Schweser, F., Deistung, A., Lehr, B.W., Reichenbach, J.R., 2011. Quantitative imaging of intrinsic magnetic tissue properties using MRI signal phase: an approach to in vivo brain iron metabolism? Neurolmage 54, 2789-2807. http://dx.doi.org/10.1016/j.neuroimage.2010. 10.070 .

Schweser, F., Sommer, K., Deistung, A., Reichenbach, J.R., 2012. Quantitative susceptibility mapping for investigating subtle susceptibility variations in the human brain. Neurolmage 62, 2083-2100. http://dx.doi.org/10.1016/j.neuroimage.2012.05.067.

Schweser, F., Deistung, A., Sommer, K., Reichenbach, J.R., 2013. Toward online reconstruction of quantitative susceptibility maps: superfast dipole inversion. Magn. Reson. Med. 69, 1582-1594.

Shmueli, K., de Zwart, J.A., van Gelderen, P., Li, T.-Q., Dodd, S.J., Duyn, J.H., 2009. Magnetic susceptibility mapping of brain tissue in vivo using MRI phase data. Magn. Reson. Med. 62 , 1510-1522. http://dx.doi.org/10.1002/mrm.22135.

Shmueli, K., Dodd, S.J., Li, T.-Q., Duyn, J.H., 2011. The contribution of chemical exchange to MRI frequency shifts in brain tissue. Magn. Reson. Med. Off. J. Soc. Magn. Reson. Med. Soc. Magn. Reson. Med. 65, 35-43. http://dx.doi.org/10.1002/mrm.22604.

Wharton, S., Bowtell, R., 2010. Whole-brain susceptibility mapping at high field: a comparison of multiple- and single-orientation methods. Neurolmage 53, 515-525. http://dx doi.org/10.1016/j.neuroimage.2010.06.070.

Wharton, S., Bowtell, R., 2013. Gradient echo based fiber orientation mapping using R2* and frequency difference measurements. Neurolmage 83, 1011-1023. http://dx.doi.org/10. 1016/j.neuroimage.2013.07.054.

Wu, B., Li, W., Guidon, A., Liu, C. 2012. Whole brain susceptibility mapping using compressed sensing. Magn. Reson. Med. 67, 137-147. http://dx.doi.org/10.1002/mrm.23000.

Yablonskiy, D.A., Luo, J., Sukstanskii, A.L., Iyer, A., Cross, A.H., 2012. Biophysical mechanisms of MRI signal frequency contrast in multiple sclerosis. Proc. Natl. Acad. Sci. 109, 14212-14217. http://dx.doi.org/10.1073/pnas.1206037109.

Zhong, K., Leupold, J., von Elverfeldt, D., Speck, O., 2008. The molecular basis for gray and white matter contrast in phase imaging. Neurolmage 40,1561-1566. http://dx.doi. org/10.1016/j.neuroimage.2008.01.061.

Zhou, J., Golay, X., van Zijl, P.C., Silvennoinen, M.J., Kauppinen, R., Pekar, J., Kraut, M., 2001 Inverse $\mathrm{T}(2)$ contrast at 1.5 Tesla between gray matter and white matter in the occipital lobe of normal adult human brain. Magn. Reson. Med. Off. J. Soc. Magn. Reson. Med. Soc. Magn. Reson. Med. 46, 401-406. 